\title{
Circuit
}

Musiques contemporaines

\section{Introduction. Prélude non mesuré}

\section{Maxime McKinley}

Volume 28, numéro 2, 2018

Instrumentarium baroque : précédence et créativité

URI : https://id.erudit.org/iderudit/1051288ar

DOI : https://doi.org/10.7202/1051288ar

Aller au sommaire du numéro

Éditeur(s)

Circuit, musiques contemporaines

ISSN

1183-1693 (imprimé)

1488-9692 (numérique)

Découvrir la revue

Citer ce document

McKinley, M. (2018). Introduction. Prélude non mesuré. Circuit, 28(2), 5-9. https://doi.org/10.7202/1051288ar

Ce document est protégé par la loi sur le droit d'auteur. L'utilisation des services d'Érudit (y compris la reproduction) est assujettie à sa politique d'utilisation que vous pouvez consulter en ligne.

https://apropos.erudit.org/fr/usagers/politique-dutilisation/
Cet article est diffusé et préservé par Érudit.

Érudit est un consortium interuniversitaire sans but lucratif composé de l’Université de Montréal, l'Université Laval et l'Université du Québec à Montréal. Il a pour mission la promotion et la valorisation de la recherche. https://www.erudit.org/fr/ 


\section{Introduction. Prélude non mesuré}

Maxime McKinley

Une anecdote fait sourire les historiens et les amateurs d'art depuis plus d'un siècle maintenant. En 1908, le Douanier Rousseau aurait déclaré à Picasso, lors d'un banquet: "Nous sommes les deux plus grands peintres de notre temps, toi, dans le genre égyptien, moi, dans le genre moderne'. » Cette histoire, bien que surtout amusante, n'est pas pour autant dépourvue d'une certaine profondeur. Il n'est pas inexact de dire que la peinture de Picasso, certes plus moderne aux yeux de l'histoire de l'art que celle du Douanier Rousseau, prend néanmoins appui sur des pratiques très anciennes, voire archaïques. C'est ainsi qu'à partir de ce mot d'esprit apparemment ingénu du Douanier Rousseau, s'apparentant à une boutade, beaucoup pourrait être dit sur le rapport de l'art au temps. On pense, par exemple, au très beau livre Devant le temps. Histoire de l'art et anachronisme des images de Georges Didi-Huberman². Un ciel étoilé de Fra Angelico (vers 1400-1455) et un allover de Jackson Pollock (1912-1956) auraient-ils plus en commun qu'il n'y paraît de prime abord? Si le passé est perçu depuis le temps présent comme point de fuite, peut-être serait-il possible de parler de synchronisme? Ce qu'il nous reste de Fra Angelico nous serait ainsi contemporain, tout en nous précédant. C’est précisément afin de prendre en compte cette perspective résolument présente sur l'ancien que le titre de ce numéro fait appel au mot "précédence » plutôt que "passé »3. Du reste, une figure aussi incontournable de la mise au jour de l'instrumentarium baroque que Nikolaus Harnoncourt affirmait sans ambages qu'« on ne peut pas faire tourner la roue de l'Histoire à l'envers ${ }^{4} »$. Cela est d'autant plus vrai pour nous, dans le cadre de ce numéro de Circuit, que ce n'est guère le débat sur l'authenticité (ou non) des interprétations qui nous intéresse ici, mais bien l'enjeu de la créativité - même «absolument moderne ${ }^{5} »-$ qui, sans imitation fétichiste du passé, « rebondit»
1. Cette anecdote fut relatée, notamment, dans cet article du journal Le Monde, le $1^{\mathrm{er}}$ mars 2010 : http://www.lemonde.fr/culture/ article/2010/03/01/henri-rousseaudecidement-moderne_1312219_3246. html (consulté le 20 avril 2018).

2. Paris, Minuit, 2000. Voir aussi : Giorgio Agamben (2008), Qu'est-ce que le contemporain?, Paris, Rivages.

3. Dans un article de 2015 intitulé "L'Affaire Novalis et l'idée de la précédence", le poète Philippe Beck cite une formule de Novalis en exergue: "Un moi ne commence pas." Voir https://www.sitaudis.fr/ Incitations/l-affaire-novalis-et-I-ideede-la-precedence.php (consulté le 20 avril 2018). Beck évoque aussi cette idée du "moi précédé" dans son art poétique Contre un Boileau (2015), Paris, Fayard, p. 52.

4. Nikolaus Harnoncourt ([1982]1984), Le Discours musical, Paris, Gallimard, p. 105.

5. Allusion au célèbre "Il faut être absolument moderne" d'Une saison en enfer: Arthur Rimbaud ([1873]1993), Poésies, Paris, Phidias, p. 146. 
6. Estimées à environ 18000 ans

7. En musique, Boris de Schloezer et Marina Scriabine contestaient déjà cette idée dans leur livre à quatre mains Problèmes de la musique moderne (1959), réédité et présenté par Bernard Sève en 2016 aux Presses universitaires de Rennes (voir le passage sur le progrès en art, p. 92).

8. Ce qui est la posture d'une importante partie du romantisme allemand, telle qu'elle est décrite par Carl Dahlhaus dans L'idée de la musique absolue. Une esthétique de la musique romantique ([1978]1997), Genève, Contrechamps.

9. Lydia Goehr ([1992]2002), The Imaginary Museum of Musical Works. An Essay in the Philosophy of Music, Oxford, Oxford University Press.

10. Voir, par exemple, Harnoucourt, op. cit.: "[...] Cherubini mit un terme à l'ancienne relation maître-apprenti au Conservatoire. II fit écrire par les plus grands noms de l'époque des ouvrages d'enseignement qui devaient réaliser dans la musique le nouvel idéal d'égalité. [...] Les professeurs les plus importants en France durent consigner les nouvelles idées sur la musique dans un système rigide. [...] Mais ce qui me paraît franchement grotesque c'est que ce système soit aujourd'hui encore la base de notre éducation musicale! Tout ce qui auparavant avait de l'importance a été ainsi anéanti " (p. 29)

11. Voir, par exemple, André Schaeffner ([1936]1994), L'origine des instruments de musique, Paris, Éditions de l'EHESS. On trouvera aussi d'enrichissantes informations et réflexions dans Bernard Sève (2013), plutôt sur lui. Si peu d'historiens, à la vue de magnifiques peintures rupestres du paléolithique, comme celles de la grotte de Lascaux ${ }^{6}$, maintiennent l'idée d'un véritable progrès en art (au sens d'une amélioration linéaire au fil des $\left.\hat{a ̂ g e s}^{7}\right)$, cela ne disqualifie en rien les notions de changements, de transformations et de nouveautés. La précédence ainsi comprise (loin de tout projet plus ou moins réactionnaire de restauration et de refus de l'avenir) rappelle, par ailleurs, que les œuvres n'apparaissent pas ex nihilo de quelque génie créateur (sorte d'Individu Suprême) ne devant rien à personne, comme un cheveu sur la soupe. Il y a du commun, du collectif, dans l'histoire et le savoir, et cette dimension échapperait à une conception de l'œuvre d'art réduite à un objet totalement autocentré et « détaché8 ${ }^{8}$. C'est ainsi qu’une théoricienne comme Lydia Goehr, par exemple, a choisi de penser la musique davantage en termes historiques qu'en termes d'œuvres?.

En matière d'art, nous retrouvons souvent, dans la doxa moderniste, un rapport à la beauté privilégiant l'avers des conventions sociales de celle-ci, dans une sorte de célébration de l'inconfort - ou, plus précisément, dans une certaine méfiance du confort. Mais à quoi correspondrait le confort dans la musique de tradition dite «savante » occidentale? Peut-être à ce qui n'est plus questionné à la racine: l'habituel, le réflexe, ce qui va supposément de soi et qui, ainsi, rétrécit le champ des possibles. À ce titre, une ligne est parfois tracée par les historiens autour de 1800. C'est-à-dire qu’après la Révolution française, avec l'institutionnalisation du Conservatoire et les traités rédigés sous l'impulsion de Cherubini, plusieurs aspects des pratiques musicales de cette tradition se seraient quelque peu uniformisés, sinon figés ${ }^{10}$. Un endroit où certains compositeurs de musiques dites «contemporaines» et certains instrumentistes dits «baroqueux» se rejoignent, peut-être, serait dans un désir commun de sortir de ces cadres préconçus et stables (voire sclérosés).

À cet égard, voici - brossées à grands traits - quelques préoccupations contemporaines qui résonnent (de manières plus ou moins tendues) avec certaines recherches théoriques et sonores des XVII et XVIII ${ }^{e}$ siècles. D'Helmut Lachenmann à Jean-François Laporte (pour ne citer que deux compositeurs d'aujourd'hui), l'enjeu de la production même du son a été repensé et travaillé radicalement (du côté des modes de jeu dans le premier cas, et de l'invention d'instruments dans le second). Sans forcer exagérément le parallèle, on constate, en étudiant l'organologie, à quel point la lutherie et la technique de plusieurs instruments n'étaient pas encore stabilisées à l'époque baroque, ce qui permettait un rapport au sonore très ouvert en termes de possibilités ${ }^{11}$. D'Ivan Wyschnegradsky à Georg Friedrich Haas, en passant par Bruce Mather et Pascale Criton (entre autres), beaucoup de 
compositeurs, depuis quelques décennies, ont voulu assouplir (ou élargir) le cadre du tempérament égal à douze sons par des micro-intervalles ${ }^{12}$. À ce sujet, nous savons de plus en plus à quel point la question de l'intonation et des tempéraments est extrêmement riche et variée à travers l'histoire de la musique, particulièrement avant la standardisation du tempérament égal, que d'aucuns ont très tôt considéré comme étant bien pauvre (désagréable, même) comparativement à d'autres possibilités ${ }^{13}$. Nous pourrions encore citer comme exemple les recherches sur la mise en espace du son, de Xenakis aux orchestres de haut-parleurs, en passant par le collectif Espaces sonores illimités ${ }^{14}$, notamment. La prise en compte et l'utilisation de l'espace et des spécificités acoustiques d'un lieu précis existaient bien avant l'art in situ contemporain $^{15}$, et menaient il y a quelques siècles à des possibilités de présentations différentes du concert habituel que nous connaissons aujourd'hui. La liste pourrait continuer de s'allonger, mais ne citons qu'un dernier exemple. Dès les années 1950, les recherches sur la notation musicale et les «œuvres ouvertes $^{16}$ » donnant un plus grand rôle créatif aux interprètes se sont multipliées, de Boulez, Stockhausen et Pousseur à Brown, Cage et Feldman, en passant par Boucourechliev et bien d'autres. Quant au rôle créatif joué par les interprètes dans la musique baroque, il est bien connu que les compositeurs de cette époque étaient souvent eux-mêmes de brillants instrumentistes et improvisateurs, et que leurs notations musicales étaient généralement sciemment « lacunaires » (préludes non mesurés, basses chiffrées, ornementations, instrumentations, nuances et articulations non spécifiées), laissant aux musiciens de nombreuses possibilités d'interprétations ${ }^{17}$. Ces exemples montrent combien le «poussiéreux » est, parfois, porteur de modernitée ${ }^{18} \ldots$

Tout ceci étant posé, une nuance s'impose. Un compositeur est habituellement un mélomane (l'inverse est difficile à imaginer) et peut, en tant que mélomane, s'intéresser à tout cela sans pour autant passer à l'acte quant à l'utilisation, pour ses propres œuvres, d'instruments dits d'époque. En fait, cela reste plutôt rare, bien qu'une liste exhaustive des œuvres contemporaines faisant appel à des instruments anciens ne soit pas si aisée et rapide à établir. Au $\mathrm{Xx}^{\mathrm{e}}$ siècle, parmi les œuvres de cet acabit ayant fait date, on pense, par exemple, à Musik für Renaissance Instrumente (1965-1966) de Mauricio Kagel, à Continuum (1968) de György Ligeti, pour clavecin, ou encore à l'opéra Medeamaterial (1992) de Pascal Dusapin ${ }^{19}$. Ces œuvres, sans doute intrigantes par leurs instrumentations, ne se réduisent toutefois pas à de simples curiosités et sont assez fortes pour s'être imposées dans la durée. Au Québec, deux œuvres faisant appel au clavecin ${ }^{20}$ ont aussi marqué le milieu des musiques de création : Suite baroque (1989) d’Yves Daoust (une œuvre électroacoustique)
12. Nous reviendrons d'ailleurs sur cet enjeu, dans le vol. 29, $n^{\circ} 2$ (numéro qui sera codirigé par Robert Hasegawa et Paul Bazin).

13. Voir Ross W. Duffin (2007), How Equal Temperament Ruined Harmony (and Why You Should Care), New York et London, Norton.

14. Voir http://www.

espacessonoresillimites.com/ (consulté le 20 avril 2018).

15. Sujet qui fut abordé dans notre numéro "Musique in situ" (vol. 17, $\mathrm{n}^{\circ} 3$, 2007).

16. Rappelons le classique d'Umberto Eco : L'OEuvre ouverte ([1962]1965), Paris, Seuil.

17. En prenant en compte, certes, une tradition orale et des conventions établies (notamment dans divers traités, très variés selon les régions) qui avaient beaucoup d'importance.

18. Cela apparaît surtout en creusant quelques points de recherche, mais il arrive occasionnellement que même à l'écoute immédiate, des œuvres baroques rejoignent étonnamment des sonorités associables à l'après-guerre, le cas le plus célèbre étant sans doute le mouvement "Le Chaos" dans Les Éléments (1737) de Jean-Féry Rebel.

19. Peu de documentation liée au thème du présent numéro semble être disponible à ce jour, mais signalons ici l'article de Jonathan Nubel "Le timbre baroque dans Medeamaterial de Pascal Dusapin". http://www.musimediane. com/numero7/NUBEL/index.html (consulté le 20 avril 2018). Ce numéro 7 de la revue Musimédiane, paru en septembre 2013, est intitulé "Le timbre baroque". Notons au passage que notre vol. 29, $n^{0} 1$ sera par ailleurs consacré à Pascal Dusapin.

20. Il s'agit d'ailleurs, dans les deux cas, de projets en collaboration avec la claveciniste et animatrice Catherine Perrin. 
21. L'œuvre, sur un livret du compositeur, relate l'histoire de Wanda Landowska (1879-1955), qui a grandement contribué à la revitalisation du clavecin au $\mathrm{Xx}^{\mathrm{e}}$ siècle.

22. À noter qu'on peut trouver ces œuvres de Beaupré et Daoust sous étiquette empreintes DIGITALes (IMED0160 et IMED-9106 respectivement).

23. D’autres musiciens établis à Montréal ont commandé des œuvres pour instruments baroques, notamment Luc Beauséjour et Matthias Maute (lui-même compositeur).

24. Les instruments baroques, pour livrer les merveilles inimitables qu'ils ont à offrir - y compris, voire surtout, les nouvelles -, demandent tout particulièrement d'être compris et considérés dans leurs spécificités. La proximité avec l'instrument et une collaboration étroite avec l'interprète sont souvent, en ce sens, cruciales.

25. Voir https://revuecircuit.ca/web/ (consulté le 20 avril 2018). ainsi que Sacrée Landowska, mémoires modernes d'outre-tombe (2001) de John Rea (un théâtre musical21). Mentionnons, en outre, Humeur de facteur (19981999), une œuvre électroacoustique composée par le facteur de clavecins Yves Beaupré, et dont la démarche est assez inusitée : tout ce qu'on y entend provient de prises de son faites dans son atelier ${ }^{22}$. Enfin, pensons, sur la scène montréalaise des musiques de création improvisées, à Katelyn Clark (organetto, clavecin), Terri Hron (flûtes à bec), Pierre-Yves Martel (viole de gambe) ou encore Jennifer Thiessen (viole d'amour).

Le paysage quelque peu bigarré esquissé jusqu’ici pourrait, bien sûr, être longuement approfondi et précisé. Le présent numéro de Circuit propose quelques «études de cas» détaillées de ce type d’approches. Ces «cas» sont géographiquement variés (avec des compositeurs du Canada, de Chypre, des États-Unis, de la France et de la Suède), et sont tous bien ancrés dans le $\mathrm{xxI}^{\mathrm{e}}$ siècle. Ainsi, dans l'article ouvrant le numéro, la violoncelliste américano-canadienne Elinor Frey nous fait découvrir un projet de six commandes d'œuvres pour divers types de violoncelles baroques ${ }^{23}$. À travers les œuvres d'Isaiah Ceccarelli, Scott Godin, Linda Catlin Smith, Lisa Streich, Ken Ueno et moi-même, Frey nous fait découvrir le(s) violoncelle(s) sous un angle à la fois nouveau et ancien ${ }^{24}$. À la suite de cet article, les univers de trois compositeurs français sont abordés tour à tour. D’abord, Laurent Feneyrou nous convie à une plongée aussi fascinante qu'érudite dans des œuvres faisant appel à des instruments (incluant la voix) baroques de Brice Pauset - qui est non seulement compositeur, mais aussi interprète (clavecin, piano) en plus d'avoir étudié la fabrication d'instruments anciens et la philosophie médiévale. Le fil de l'interprétation n'est pas rompu dans l'article suivant, puisque la claveciniste et musicologue Adèle Gornet y signe une étude fouillée d'une œuvre pour clavecin de Gérard Pesson: un Tombeau de Rameau, composé en 2014 dans le cadre du $250^{\mathrm{e}}$ anniversaire du décès de ce dernier. Notons d'ailleurs, au registre des anniversaires, que cet article est aussi une manière, pour Circuit, de célébrer les 60 ans de Pesson (ce ravélien admirateur de Lachenmann étant né en 1958). Puis, François-Xavier Féron, membre de notre comité de rédaction, aborde un enjeu à la fois complexe et intéressant en filigrane d'une œuvre de David Hudry: la confrontation d'instruments baroques et «modernes » dans une même partition. En contrepoint à ce texte riche en propos cités du compositeur, on trouvera, dans nos Suppléments $w_{e b}{ }^{25}$, des extraits sonores de l'œuvre commentés par ce dernier. Enfin, ce dossier thématique se déplace vers l'Est, plus précisément à Chypre, avec un texte du compositeur Evis Sammoutis dans lequel est relaté un projet de « recherche-création » où les manuscrits anciens, les archets baroques, le 
violon et les nouvelles technologies se rencontrent. Si contrastées soient-elles, ces démarches ont en commun d'assumer avec profondeur une part de précédence tout en étant ardemment créatives, ces deux aspects n'agissant pas comme une aporie mais comme des vases communicants.

En complément au dossier thématique, le lecteur trouvera deux autres rubriques. Ainsi, le Cahier d'analyse, signé par Landon Morrison, est consacré à la compositrice Zosha Di Castri, née à Calgary ${ }^{26}$ et maintenant Assistant Professor of Music à la Columbia University. Bien que l'œuvre analysée soit un quatuor à cordes « modernes ${ }^{27}$ ", il ne serait peut-être pas incongru de faire un parallèle entre l'approche de Zosha Di Castri et le baroque entendu lato sensu, au sens esthétique du terme. Pensons par exemple, en ratissant large, à Gilles Deleuze dans Le Pli. Leibniz et le baroque ${ }^{28}$, ou encore au compositeur Jean Lesage qui se réclame depuis longtemps du baroque, en particulier de Frescobaldi et du stylus phantasticus (en privilégiant la fantaisie, la rupture, l'extravagance ${ }^{29}$ ). Dans le même esprit, il convient ici de dire un mot sur les images du numéro, qui sont du très grand artiste québécois René Derouin. Ces œuvres sont issues de la série Paraíso. La dualité du baroque, et c'est précisément dans un sens généreux et large que le baroque y est abordé, comme en témoignent certaines notes de l'artiste en marge de ses esquisses. Il est très intéressant également de noter que Derouin pratique, dans un art tout à fait moderne, des techniques anciennes telles que la gravure sur bois. Enfin, la rubrique Actualités est entièrement consacrée à la plus récente saison (2017-2018) de l'Ensemble contemporain de Montréal (ECM+), qui célébrait son $30^{\mathrm{e}}$ anniversaire. Le compositeur Éric Champagne y propose un regard englobant sur cet organisme essentiel à la création musicale canadienne, ponctué de quelques propos de Véronique Lacroix, sa directrice artistique et fondatrice. Marie-Pierre Brasset et Keiko Devaux nous offrent, pour leur part, des comptes rendus de deux événements marquants de cette saison anniversaire ${ }^{30}$. De plus, trois collaborateurs de longue date de l'ECM+, de trois générations différentes, partagent leurs expériences dans des témoignages aussi touchants qu'instructifs: les compositeurs Gordon Fitzell et Michel Gonneville, ainsi que la violoniste Andréa Tyniec. Quelques photographies complètent ces souvenirs du futur...

Bonne lecture!

Montréal, avril 2018
26. Et qui a grandi à Saint-Albert, en Alberta, à proximité d'Edmonton.

27. Les guillemets de précaution s'imposent ici, tant l'utilisation de cet adjectif, courante dans ce genre de contextes, est néanmoins problématique; "moderne" est relatif, dans le cas qui nous occupe, aux instruments baroques: il s'agit simplement de désigner les instruments à cordes tels qu'utilisés selon les standards d'aujourd'hui (avec des cordes faites de matériaux synthétiques plutôt qu'en boyaux, par exemple).

28. Paris, Minuit, 1988.

29. Voir Jean Lesage (1999), "La musique, le baroque et le second degré", dans Présence de la musique québécoise. Vingt-deux portraits instantanés, Denys Bouliane (dir.), Montréal, s.é., p. 51-53. Rappelons aussi le concert monographique que l'ECM+ consacra à Jean Lesage, le 26 avril 2017 à la Salle Pierre-Mercure, et justement intitulé Stylus Phantasticus.

30. Il s'agit, respectivement, du concert World New Music Days_BIS et de l'opéra d'André Ristic Hockey Noir. 